\title{
CHARMONIUM AND BOTTOMONIUM TRANSITIONS FROM EFFECTIVE FIELD THEORY
}

\author{
ULF-G. MEIßNER \\ Helmholtz Insitut für Strahlen- und Kernphysik 83 Bethe Center for Theoretical Physics \\ Universität Bonn, D-53115 Bonn, Germany \\ Institut für Kernphysik, Institute for Advanced Simulation \& Jülich Center for Hadron Physics \\ Forschungszentrum Jülich, D-52425 Jülich, Germany \\ meissner@hiskp.uni-bonn.de
}

\begin{abstract}
The effects of intermediate charmed mesons on charmonium transitions with the emission of one pion or eta meson are studied systematically. Based on a non-relativistic effective field theory we show that charmed meson loops are enhanced compared to the corresponding tree-level contributions for transitions between two $S$-wave charmonia as well as for transitions between two $P$-wave charmonia. On the contrary, for the transitions between one $S$-wave and one $P$-wave charmonium state, the loops need to be analyzed case by case and often appear to be suppressed. Extending these considerations to the $b$-quark sector, we propose a new method to extract the light quark mass ratio $m_{u} / m_{d}$ using the $\Upsilon(4 S) \rightarrow h_{b} \pi^{0}(\eta)$ bottomonia transitions.
\end{abstract}

Keywords: Charmonium transitions, quark masses, chiral Lagrangian

\section{Introduction}

Since the discovery of the $J / \psi$ more than thirty years ago, the decays of heavy quarkonia have played an important role in the physics of quarks and hadrons. In particular, they also allow for precision studies in the light quark sector. Because the $\psi^{\prime}$ and $J / \psi$ are isospin and $\mathrm{SU}(3)$ flavor singlets, the decay process $\psi^{\prime} \rightarrow J / \psi \pi^{0}$ violates isospin symmetry and $\psi^{\prime} \rightarrow J / \psi \eta$ violates $\mathrm{SU}(3)$ symmetry. Isospin symmetry can be violated by both electromagnetic (em) effects and the mass difference between the $u$ and $d$ quarks. Because the em effects are small, these two decays were used to extract the light quark mass ratio $m_{u} / m_{d}^{1,2,3}$. These works were based on the QCD multipole expansion (QCDME). The QCDME is based on the assumption that the emitted gluons are soft so that their wavelengths are much larger than the size of a heavy quarkonium. As a result, a multipole expansion similar to that in classical electrodynamics can be performed. The soft gluons then hadronize into light meson(s), for instance the pion(s) or eta, and the matrix elements may be worked out using soft pion theorems. However, this ansatz clearly misses the contribution from intermediate mesons. This can be understood as a heavy quarkonium can couple to a heavy meson and heavy anti-meson pair through the non-perturbative production 
of a light quark and anti-quark pair. It was already shown in Ref. ${ }^{4}$ that the intermediate charmed meson loops lead to large corrections to the QCDME result, that make a precise extraction from $m_{u} / m_{d}$ at present impossible. Therefore, a more general analysis of charmed meson loop effects in charmonium transitions is called for and I will outline it here. For details, we refer to Refs. ${ }^{5,6}$. Further implications of heavy meson loops in charm decays are discussed by Zhao ${ }^{7}$.

\section{Outline of the calculation}

We consider the $S S, S P$ and $P P$ transitions with the emission of one $\pi^{0}$ or one $\eta$ indicated in Fig. 1. Most of them can be analyzed in a suitably tailored EFT, that combines chiral perturbation theory with a non-relativistic approach to the charmed mesons and charmonia ${ }^{6}$.

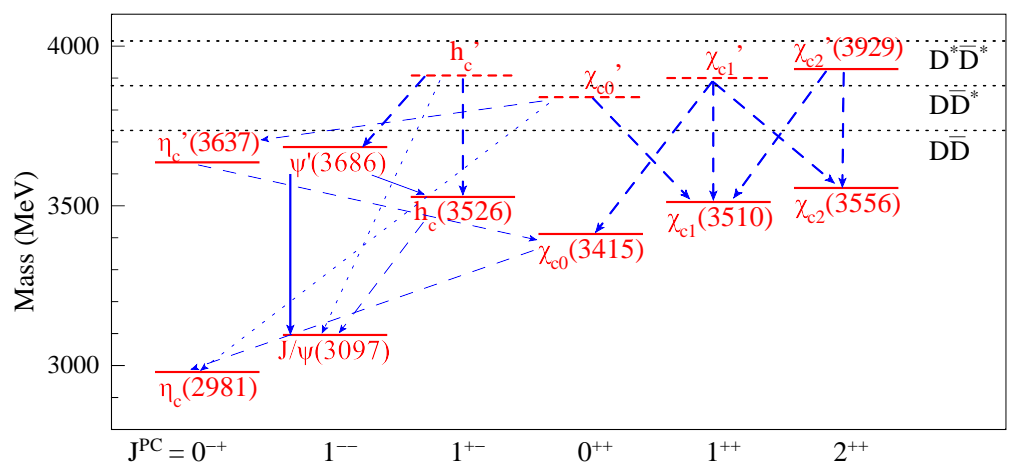

Fig. 1. All possible $S$ and $P$-wave transitions among the ground state and the first radial excited $S$ and $P$-wave charmonia with the emission of one light pseudoscalar meson $\left(\pi^{0}, \eta\right)$. The unobserved resonances and decay modes are plotted in dashed. The masses for the unobserved resonances are taken from the quark model. The thresholds for the $D \bar{D}, D \bar{D}^{*}$ and $D^{*} \bar{D}^{*}$ are represented by the dotted horizontal lines. The solid lines represent the measured decays. Unmeasured decays are shown by dashed and dotted lines, only the former are amenable to the EFT discussed here.

To leading order, we construct the tree-level chiral effective Lagrangians and calculate the resulting amplitudes are worked out for all the decays under consideration. The isospin and $\mathrm{SU}(3)$ breaking effects are given by the quark mass differences. Consistent with earlier analyses, the em contributions are found to be small and can be neglected. Next, the leading charmed meson loop corrections to all these decays are worked out. These are triangle graphs with three charmed mesons in the loop, more precisely all possible combinations of the lowest-lying pseudoscalar and vector charmed mesons. Such diagrams can be calculated using an appropriate heavy meson chiral Lagrangian as reviewed in $^{8}$. A consistent power counting that incorporates the various scales (masses, energies, scaling of the various coupling constants) can be set up and is summarized in Tab. 1. 
Table 1. Power counting of the tree-level amplitudes and the (leading) loops. Here $S S, S P$ and $P P$ represent transitions between two $S$-wave, one $S$-wave and one $P$-wave, and two $P$-wave charmonia, respectively. The parameter $\delta$ denotes the strength parameter for isospin or SU(3) symmetry violation, $v$ the heavy-meson velocity, $q$ the momentum of the outgoing pseudoscalar meson, and $M_{D}$ the mass of the heavy mesons in the loop.

\begin{tabular}{|l|c|c|c|}
\hline & $S S$ & $S P$ & $P P$ \\
\hline tree-level & $q \delta$ & $\delta$ & $q \delta$ \\
loops & $\delta \frac{1}{v} q$ & $\frac{q^{2}}{v^{3} M_{D}^{2}} \delta$ & $\delta \frac{1}{v^{3}} q$ \\
\hline
\end{tabular}

As a result of this analysis we find, comparing the loop contributions with the tree-level decay amplitudes given in Tab. 1, where we assume the same scale $\delta$ for the light quark mass and the heavy meson mass differences ${ }^{9}$, that the loop contributions for the $S S$ transitions are enhanced by a factor of $1 / v \approx 2$, and for the $P P$ transitions even by a factor of $1 / v^{3} \approx 10$. The situation for the $S P$ transitions should be analyzed case by case since an enhancement factor $1 / v^{3}$ competes with a suppression factor $q^{2} / M_{D}^{2}$, with $M_{D}$ the average D-meson mass. For the $S P$ transitions with small phase space, the external momentum $q$ might be small enough to make $q^{2} /\left(v^{3} M_{D}^{2}\right)$ much smaller than 1 , which is satisfied for the decays $\psi^{\prime} \rightarrow h_{c} \pi^{0}$ and $\eta_{c}^{\prime} \rightarrow \chi_{c 0} \pi^{05}$. In this case, the decay is dominated by the tree-level contributions. However, for the decays with external momentum $q \gtrsim M_{D} v^{3 / 2}$, the factor $q^{2} /\left(v^{3} M_{D}^{2}\right) \gtrsim 1$, so is no more a suppression, and hence the tree-level contributions are at least as important as the loop contributions. Furthermore, the power counting summarized in Tab. 1 can be shown to be explicitely consistent with the divergence structure of the pertinent one-loop integrals.

\section{Assorted results}

$S S$-transitions: The decays studied in this class are $\psi^{\prime} \rightarrow J / \psi \pi^{0}$ and $\psi^{\prime} \rightarrow J / \psi \eta$. For these transitions the charmed meson loops are enhanced by $1 / v \simeq 2$. Hence, the result for the width is dominated by the loops ${ }^{4}$, and the relatively large expansion parameter leads to a sizable uncertainty. For more details, see Ref. ${ }^{6}$.

$S P$-transitions: In these transitions, the situation is different. As shown in Tab. 1, the loops do not necessarily dominate the transitions. Especially for those decays which have a very small phase space, the contribution from the charmed-meson loops is highly suppressed ${ }^{5}$. For example, this is the case in the decays $\psi^{\prime} \rightarrow h_{c} \pi^{0}$ and $\eta_{c}^{\prime} \rightarrow \chi_{c 0} \pi^{0}$. The ratio of these two widths can be given free of any parameter:

$$
\frac{\Gamma\left(\eta_{c}^{\prime} \rightarrow \chi_{c 0} \pi^{0}\right)}{\Gamma\left(\psi^{\prime} \rightarrow h_{c} \pi^{0}\right)}=3 \frac{q_{2}}{q_{1}} \frac{M_{\chi_{c 0}} M_{\psi^{\prime}}}{M_{\eta_{c}^{\prime}} M_{h_{c}}}=5.86 \pm 0.94,
$$

where the $15 \%$ uncertainty comes from neglecting higher order terms in either the heavy quark expansion or the chiral expansion. Using the experimental value of 
$\Gamma\left(\psi^{\prime} \rightarrow h_{c} \pi^{0}\right)$, we predict the width of the $\eta_{c}^{\prime} \rightarrow \chi_{c 0} \pi^{0}$ as

$$
\Gamma\left(\eta_{c}^{\prime} \rightarrow \chi_{c 0} \pi^{0}\right)=1.5 \pm 0.3 \pm 0.2 \mathrm{keV}
$$

where the first uncertainty is experimental and the second theoretical due to neglecting higher orders. With the total width of the $\eta_{c}^{\prime}, \Gamma\left(\eta_{c}^{\prime}\right)=14 \pm 7 \mathrm{MeV}$, the branching fraction of the isospin-breaking transition is

$$
\mathcal{B}\left(\eta_{c}^{\prime} \rightarrow \chi_{c 0} \pi^{0}\right)=(1.1 \pm 0.6) \times 10^{-4} .
$$

The prediction, and therefore the dynamics underlying the decays, is testable with $\overline{\mathrm{P}} \mathrm{ANDA}$ at FAIR. More $S P$ transitions are discussed in Ref. ${ }^{6}$.

$P P$-transitions: Here, the charmed meson loops scale as $q \delta / v^{3}$. Compared to the tree-level amplitudes, the loops are enhanced by a factor of $1 / v^{3}$, see Tab. 1 . Therefore, it is reasonable to neglect the contributions from the tree-level diagrams for the transitions between two $P$-wave charmonia. There are in total seven transition processes from the first radial excited $P$-wave charmonia to the ground state ones. All of the amplitudes are proportional to the product of the same coupling constants $g g_{1} g_{1}^{\prime} / F$. Therefore, the ratios among these decay widths can be predicted without any free parameter. Stated differently, the transitions between $P$-wave charmonia, especially for the $\chi_{c J}$ decays, allow for an especially sensitive test of the meson loop transitions, A cornucopia of predictions for loop dominated decays can be found in Ref. $^{6}$.

\section{4. $m_{u} / m_{d}$ from bottomonia transitions}

The situation is markedly different in the bottomium sector: In Ref. ${ }^{10}$, we proposed as a new method to extract the light quark mass ratio to use the transitions between bottomonia, i.e., $\Upsilon(4 S) \rightarrow h_{b} \pi^{0}(\eta)$. The master formula is similar to the one for charmonia $S S$ decays discussed above and takes the form

$$
\frac{\Gamma\left(\Upsilon(4 S) \rightarrow h_{b} \pi^{0}\right)}{\Gamma\left(\Upsilon(4 S) \rightarrow h_{b} \eta\right)}=r_{G \tilde{G}}^{2}\left|\frac{\vec{q}_{\pi}}{\vec{q}_{\eta}}\right|,
$$

where $\vec{q}_{\pi(\eta)}$ is the momentum of the pion (eta) in the rest frame of the $\Upsilon(4 S)$, and the ratio of the gluon matrix elements is defined as

$$
r_{G \tilde{G}} \equiv \frac{\left\langle 0|G \tilde{G}| \pi^{0}\right\rangle}{\langle 0|G \tilde{G}| \eta\rangle} .
$$

Combining chiral perturbation theory with the $\mathrm{U}(1)_{A}$ anomaly, the next-to-leading order (NLO) expressions for the matrix elements $\left\langle 0|G \tilde{G}| \pi^{0}(\eta)\right\rangle$ were worked out in Ref. ${ }^{3}$ in terms of several low-energy constants (LECs) of the $\mathcal{O}\left(p^{4}\right)$ Lagrangian. Moreover, there exists an intriguing relation between the ratio of the matrix elements and a combination of the light quark masses ${ }^{3,11}$

$$
\begin{aligned}
R & \equiv \frac{m_{d}-m_{u}}{m_{d}+m_{u}} \frac{m_{s}+\hat{m}}{m_{s}-\hat{m}}=\frac{4}{3 \sqrt{3}} r_{G \tilde{G}} \frac{F_{\pi}}{F_{\eta}} \frac{F_{K}^{2} M_{K}^{2}-F_{\pi}^{2} M_{\pi}^{2}}{F_{\pi}^{2} M_{\pi}^{2}}\left(1-\delta_{\mathrm{GMO}}\right) \\
& \times\left[1+\frac{4 L_{14}}{F_{\pi}^{2}}\left(M_{\eta}^{2}-M_{\pi}^{2}\right)\right]=10.59\left(1+132.1 L_{14}\right) r_{G \tilde{G}},
\end{aligned}
$$


where $\hat{m}$ is the average light quark mass, $M_{\phi}\left(F_{\phi}\right)$ denotes the masses (decay constants) of the Goldstone bosons $(\phi=\pi, K, \eta), \delta_{\mathrm{GMO}}=-0.06$ represents the $\mathcal{O}\left(p^{4}\right)$ deviation from the Gell-Mann-Okubo relation among the Goldstone bosons, and $L_{14}=(2.3 \pm 1.1) \times 10^{-3}$ is a low-energy constant related to the chiral expansion of the energy-momentum tensor ${ }^{12}$. The tree level prediction for $R$ is not afflicted by large corrections from heavy meson loops. This is largely due to the extremely small B-meson mass splitting, $M_{B^{0}}-M_{B^{+}}=(0.33 \pm 0.06) \mathrm{MeV}$, which is due to an destructive interference effect between strong and electromagnetic contributions ${ }^{13}$. The estimated uncertainty of this determination of the quark mass ratio $\left(m_{d}-m_{u}\right)\left(m_{s}+\hat{m}\right) /\left(m_{d}+m_{u}\right) /\left(m_{s}-\hat{m}\right) \simeq\left(m_{d}-m_{u}\right) /\left(m_{d}+m_{u}\right)$, with $\hat{m}$ the average light quark mass, is about $23 \%$. As a side result our work identified the decay branch $\Upsilon(4 S) \rightarrow h_{b} \eta$ as a promising discovery channel for the $h_{b}$. To confirm the scheme described it is important to test the predictions from the effective field theory used also for other transitions. Especially, we predicted a large number of branching ratios for charmonium transitions. Those measurements could be performed at Belle, BES-III or PANDA.

\section{Acknowledgments}

I thank my collaborators Feng-Kun Guo, Christoph Hanhart, Qiang Zhao and Gang $\mathrm{Li}$ for sharing their insights into the topics discussed here. Work supported in part by BMBF (grant 06BN9006), DFG (SFB/TR 16), HGF (VH-VI-231) and the EU (HadronPhysics2).

\section{References}

1. B. L. Ioffe, Yad. Fiz. 29, 1611 (1979) [Sov. J. Nucl. Phys. 19, 827 (1979)].

2. B. L. Ioffe and M. A. Shifman, Phys. Lett. B 95, 99 (1980).

3. J. F. Donoghue and D. Wyler, Phys. Rev. D 45, 892 (1992).

4. F.-K. Guo, C. Hanhart and U.-G. Meißner, Phys. Rev. Lett. 103, 082003 (2009) [arXiv:0907.0521 [hep-ph]] [Erratum: ibid 104, 109901 (2010)].

5. F. K. Guo, C. Hanhart, G. Li, U.-G. Meißner and Q. Zhao, Phys. Rev. D 82, 034025 (2010) [arXiv:1002.2712 [hep-ph]].

6. F. K. Guo, C. Hanhart, G. Li, U.-G. Meißner and Q. Zhao, arXiv:1008.3632 [hep-ph].

7. Q. Zhao, these proceedings.

8. R. Casalbuoni et al., Phys. Rept. 281, 145 (1997) [arXiv:hep-ph/9605342].

9. F.-K. Guo, C. Hanhart, S. Krewald and U.-G. Meißner, Phys. Lett. B 666, 251 (2008) [arXiv:0806.3374 [hep-ph]].

10. F. K. Guo, C. Hanhart and U.-G. Meißner, Phys. Rev. Lett. 105, 162001 (2010) [arXiv:1007.4682 [hep-ph]].

11. J. F. Donoghue, B. R. Holstein and D. Wyler, Phys. Rev. Lett. 69, 3444 (1992).

12. J. F. Donoghue and H. Leutwyler, Z. Phys. C 52, 343 (1991).

13. F.-K. Guo, C. Hanhart and U.-G. Meißner, JHEP 0809, 136 (2008). [arXiv:0809.2359 [hep-ph]]. 